\title{
Gradhiva
}

Revue d'anthropologie et d'histoire des arts

17 | 2013

L'esthétique du geste technique

\section{De la beauté du geste technique en préhistoire}

A prehistory of skilled labour

\section{Sophie A. de Beaune}

\section{(2) OpenEdition \\ Journals}

Édition électronique

URL : http://journals.openedition.org/gradhiva/2583

DOI : $10.4000 /$ gradhiva.2583

ISSN : 1760-849X

\section{Éditeur}

Musée du quai Branly Jacques Chirac

\section{Édition imprimée}

Date de publication : 16 mai 2013

Pagination : 26-49

ISBN : 978-2-35744-049-74

ISSN : 0764-8928

\section{Référence électronique}

Sophie A. de Beaune, «De la beauté du geste technique en préhistoire », Gradhiva [En ligne], 17 | 2013, mis en ligne le 27 mai 2013, consulté le 30 avril 2019. URL : http://journals.openedition.org/ gradhiva/2583 ; DOI : 10.4000/gradhiva.2583 



\section{De la beauté du geste technique en préhistoire}

par Sophie A, de Beaune

Quelles relations les outils de la préhistoire entretiennent-ils entre esthétique et fonction? À partir de l'examen d'un objet qui présente la particularité de disposer de deux plans de symétrie perpendiculaires, le biface, la notion leroi-gourhanienne d'esthétique fonctionnelle est ici revisitée. L'auteur propose I'hypothèse que le geste qui se cache derrière l'outil est lui aussi susceptible de receler une certaine beauté, qui n'est pas étrangère à l'esthétique des objets eux-mêmes. 
1. Je me permets de renvoyer ici à Beaune 1999 (1995) et 2013 (2007).

\section{Sahlins 1972.}

3. Le Paléolithique supérieur s'étend d'environ 40000 à 12000 ans avant le présent. Il est le fait de l'homme anatomiquement moderne, Homo sapiens.

4. Les termes "beau " et "esthétique " seront définis au fur et à mesure de la progression de ma réflexion.

5. Sur les aptitudes cognitives des premiers homininés de la préhistoire, voir Beaune 2009 et 2011. L'étude de l'émergence des tout premiers outils ainsi que des processus de transfert technique tout au long de la préhistoire montre que, sans aller jusqu'à poser l'existence d'universaux biologiques et cognitifs communs à tout le genre Homo (voire aux australopithèques fabricants d'outils), les aptitudes cognitives nécessaires à l'invention étaient présentes très tôt.
"L'homme a toujours eu l'amour du beau. Même aux temps primitifs, où les besoins de la vie matérielle absorbaient tous ses moments, il cherchait à rendre ses armes et ses outils symétriques et à leur donner une forme élégante. » Édouard Piette (1889: 159)

«Dès qu'apparaît la plastique, on voit surgir des notions d'équilibre, donc des notions de rythme; et dès qu'apparaît la rythmique, l'art apparaît. Socialement et individuellement, l'homme est un animal rythmique. » Marcel Mauss (1967 [1947]: 85)

L'image de l'homme de la préhistoire est ambivalente. II est vu tantôt comme un pauvre hère vêtu «de peaux de bêtes,/échevelé, livide au milieu des tempêtes... ", tantôt comme un ingénieux artisan capable d'adapter parfaitement ses outils à ses besoins, presque un designer avant la lettre. Si la première de ces images fait encore les beaux jours de la littérature ou du cinéma de vulgarisation, nos connaissances sur le mode de vie des hommes de la préhistoire l'ont aujourd'hui frappée d'obsolescence. Les spécialistes savent désormais que les chasseurs-cueilleurs du Paléolithique supérieur étaient parfaitement intégrés à leur environnement et suffisamment bien organisés pour être dispensés de consacrer l'essentiel de leur temps à se procurer de quoi survivre ${ }^{\mathbf{1}}$. On peut dire que, comme les chasseurs-cueilleurs contemporains, dont l'image a été réhabilitée en particulier par Marshall Sahlins ${ }^{2}$, les groupes humains préhistoriques - du moins ceux vivant au Paléolithique supérieur ${ }^{3}$, mieux connus - vivaient dans une relative aisance et étaient beaucoup moins besogneux qu'on ne l'avait longtemps cru. Le temps passé à l'acquisition et à la préparation de la nourriture n'excédait sans doute pas chez eux cinq heures par jour, ce qui leur laissait assez de temps pour se consacrer à l'art, au jeu, à la musique et aux devoirs de la vie sociale ou religieuse.

La question de l'adaptation de l'homme préhistorique à son milieu étant réglée - nous sommes du reste la preuve vivante de cette excellente adaptation -, nous allons explorer ici plus en détail la seconde de ces images et évaluer dans quelle mesure la beauté que nous trouvons à certains outils de la préhistoire a été recherchée par leurs créateurs. En d'autres termes, quelle part leur souci d'efficacité technique laissait-il aux préoccupations esthétiques ${ }^{4}$ ? Mais précisons d'emblée que les outils à la forme régulière et symétrique dont il va être question ici sont bien plus anciens que le Paléolithique supérieur. Ils apparaissent pour la première fois en Afrique il y a près de deux millions d'années. Leur premier auteur est un lointain précurseur d'Homo sapiens. D'où la question de savoir s'il est légitime d'attribuer des préoccupations esthétiques aux premiers représentants du genre Homo - habilis, erectus, rudolfensis, neandertalensis... La réponse à cette question me paraît devoir être positive. Tous fabriquaient des outils à partir de techniques de débitage et de façonnage de la pierre déjà complexes qui impliquaient une bonne maîtrise de leur environnement, une excellente habileté technique et l'aptitude au raisonnement analogique ${ }^{5}$. Et tous - qu'ils soient en Afrique, en Europe ou en Asie - ont manifesté le même souci de régularité et de symétrie dans la fabrication de leurs outils, ou du moins de celui dont je parlerai. 


\section{Beauté des objets techniques}

Venons-en donc à notre outil, le biface, très simple, très ancien, constitué de deux faces qui lui ont valu son nom. Elles sont de surcroît symétriques l'une de l'autre et chacune d'elles est pourvue d'un plan de symétrie, ce qui fait pour l'ensemble de l'outil deux plans de symétrie perpendiculaires (fig. 1). Les préhistoriens, dont nous verrons qu'ils en ont jugé un peu vite, se sont longtemps accordés à lui trouver des formes parfaitement adaptées à sa fonction. En même temps, ils aiment à penser - et, sur ce point, je ne leur donne pas tort - que la symétrie de ces formes témoigne d'une recherche esthétique. Nous devrions donc considérer les fabricants de bifaces comme de parfaits designers puisque, selon l'une de ses définitions les plus communes, le design consiste en une recherche d'harmonie entre les formes et les fonctions d'un objet. Du reste, certains designers se réfèrent explicitement à ces lointains devanciers. Ainsi, les «couteaux de céramique » imaginés par le New-Yorkais Matthias Kaeding se réclament du Néolithique, sans doute à cause de leur forme censée évoquer celle des lames de hache polies ${ }^{6}$. De même, voici comment Marie Garnier commente un objet de «design culinaire» qu'elle a baptisé «Homo sapiens» (fig. 2):

Homo sapiens: le premier outil préhistorique pour l'homme moderne! Transformer la matière culinaire, aiguiser une lame d'acier avec une pierre taillée... Des gestes simples et intuitifs qui nous rappellent les premiers gestes de l'homme et établissent un lien entre le présent et le passé. Ce caillou sculpté (grès) est un véritable «bijou de cuisine » qui répond à plusieurs fonctions: aiguiser les couteaux mais aussi concasser, broyer des grains de poivre ou de l'ail... Conçu pour être suspendu à un crochet, il se range facilement tout en décorant la pièce de sa présence insolite ${ }^{7}$.

Ces artistes ne font que redire à leur manière ce qu'André LeroiGourhan écrivait déjà dans Le Geste et la Parole. Le fait que tel ou tel outil (biface, racloir, couteau...), «très adapté à son usage particulier, laisse transparaître à un égal degré la qualité esthétique de la rencontre de la fonction et de la forme" relève de ce qu'il appelle une "esthétique fonctionnelle». Pour lui, la valeur esthétique d'un outil varie en directe raison de l'adéquation de sa forme à sa fonction (Leroi-Gourhan 1965: 120 sq.). Cette beauté «fonctionnelle ${ }^{8}$ » serait présente dans toutes les productions humaines dès lors que leur forme s'est dépouillée de tout superflu et ne matérialise plus que leur seule fonction. Ainsi, le poinçon, qu'il soit en pierre taillée ou en acier, est mécaniquement parfait; on pourra donc le dire beau, au même titre, par exemple, que les alvéoles des abeilles ou l'aile de l'albatros. De même, il existe une grande variété de couteaux, dont chacune est susceptible d'atteindre une forme idéale - et donc belle - correspondant à l'adéquation parfaite à sa fonction: un couteau lisse ou un couteau à dents n'ont pas le même usage, et la beauté du premier ne peut être celle du second.

Leroi-Gourhan remarque cependant que, si nous sommes aujourd'hui portés à placer la beauté du côté des outils dont les formes sont les plus "pures", nombreuses sont les cultures où les formes «parfaites» ont été considérées comme pauvres. II oppose par exemple «la lame de sabre
6. «Back to basics: design "néolithique" en cuisine"; http://sleekdesign.canalblog. com, archive du 19 juillet 2009.

7. Http://madeindesign.com, rubrique «Ustensiles de cuisine ": "pierre à aiguiser».

8. Leroi-Gourhan 1965: 126. Selon les critères d'une telle esthétique, est beau ce qui est adapté à sa fonction. Nous sommes là à l'opposé de ce qu'on pourrait appeler une esthétique kantienne, qui tiendrait pour beau ce qui se destine à la contemplation désintéressée. Mais tout le propos du présent article est précisément de montrer que, au moins lorsqu'on parle de la beauté d'objets techniques, l'opposition n'est bien souvent qu'apparente. Sur la question, plus vaste, des rapports entre esthétique et technique, je me permets de signaler la parution récente d'un dossier dans la Revue de synthèse (Beaune et Hilaire-Pérez 2012). 


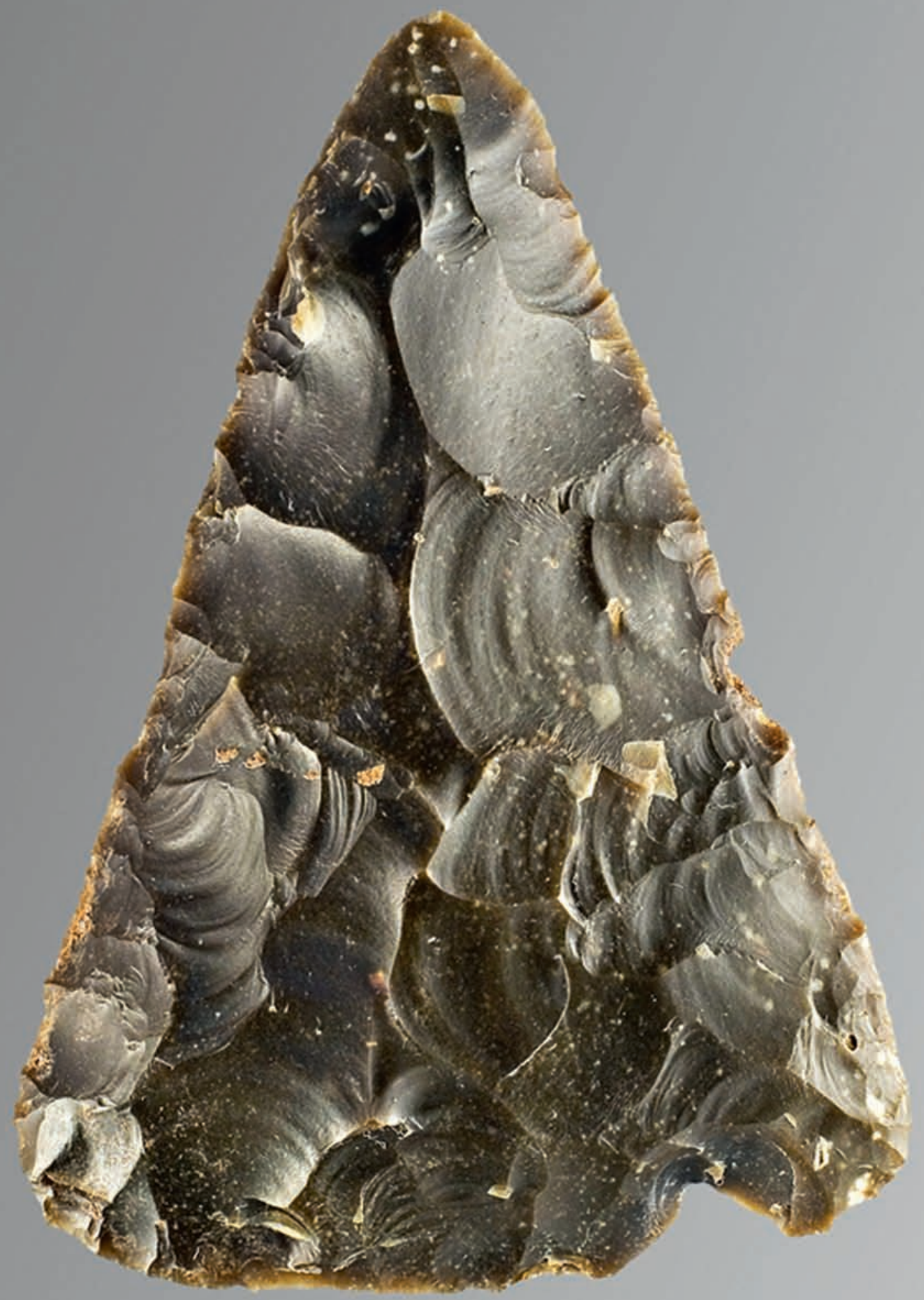




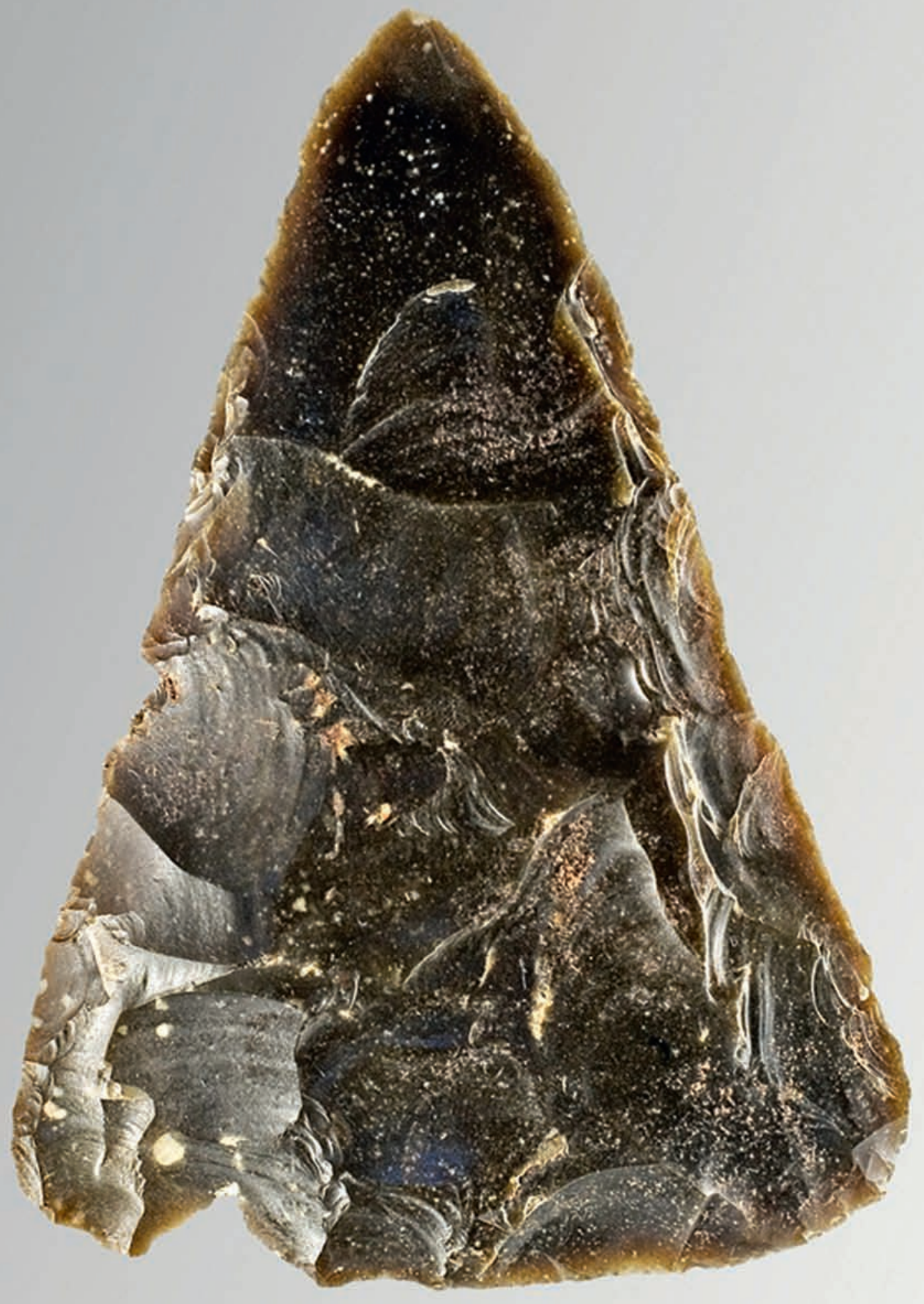


9. Les notions de tendance et de fait de Leroi-Gourhan ont donné lieu à une littérature très abondante depuis des décennies. Je ne citerai ici pour mémoire que les actes d'un colloque consacré à ce thème parus en 1994 dans la revue Techniques \& Culture (Martinelli 1994).

10. Qu'un même objet technique ait été conçu et ait évolué de la même manière chez des groupes humains éloignés dans le temps et l'espace n'a rien de curieux si l'on songe que, plus le geste technique et l'outil sont simples, plus la probabilité de la convergence est forte. À l'inverse, plus l'enchaînement des gestes est complexe, plus la diversité des outils est grande et plus la probabilité de la convergence est faible. La diversité des gestes techniques et des produits obtenus est ainsi de plus en plus grande au fu et à mesure que l'on avance sur une «lignée » technique (Beaune 2008).

11. Aristote, Politique, 1333 a 30 ff, 1332b 32, cité par Arendt 1961 (1958) : 48.

\section{double page \\ précédente}

fig. 1

Biface triangulaire provenant de l'atelier de débitage du Paléolithique moyen de Saint-Amand-les-Eaux (Nord), 2007. Archéologue responsable d'opération: Philippe Feray.

(C) Dominique Bossut, Inrap.

\section{ci contre}

fig. 2

Objet de design culinaire en grès baptisé Homo Sapiens par son auteur, Marie Garnier, édité chez ENOstudio (www.enostudio.net). japonaise [qui] est un miracle d'équilibre fonctionnel» aux armes de la Chine, de l'Inde et de l'Indonésie, qui "fourmille[nt] de formes tourmentées dans lesquelles la fonction s'étouffe sous des appendices et des courbes destinées à faire terrible" (ibid.: 128). Nos préférences actuelles seraient récentes, liées à la prépondérance des sciences mathématiques et physiques dans notre civilisation (ibid.). Est-ce si sûr? Son exemple du sabre japonais montre que les choses ne sont pas si simples. Après tout, on se rappelle que $\mathrm{M}^{\mathrm{m}}$ de Pompadour, qui, que je sache, n'était pas spécialement portée sur les mathématiques, déplorait déjà les excès du rococo en adjurant «que l'on ne tourmente plus ce qui peut être droit ».

II n'en demeure pas moins que la luxuriance fonctionnellement gratuite du baroque tardif ou des armes chinoises n'est jamais qu'une illustration extrême de situations répandues. Au côté d'une beauté tenant à une parfaite adaptation à leur fonction, les outils présentent fréquemment des variations de forme que l'on ne peut attribuer à des raisons techniques. Constat que Leroi-Gourhan exprime en distinguant la «tendance» et le «fait». Les outils et, d'une manière générale, les phénomènes techniques - gestes, chaînes opératoires, savoir-faire - sont soumis à une tendance sourde et diffuse, à laquelle il attribue une inexorabilité comparable à celle des lois de la nature. II distingue différents «degrés de fait", dont les premiers sont liés à cette tendance abstraite, prévisible et inévitable, et les derniers, imprévisibles et souvent liés au milieu culturel, incluent les variations de style opératoires et esthétiques ${ }^{9}$. On voit la tendance à l'œuvre lorsque des peuples sans liens les uns avec les autres apportent des solutions identiques à un même problème technique - les archéologues parlent ici de "convergence technique ${ }^{\mathbf{1 0}}$ ». Antoine de Saint-Exupéry pensait sans doute à une tendance lourde de cette sorte quand il écrivait:

II semble que tout l'effort industriel de l'homme, tous ses calculs, toutes ses nuits de veille sur les épures, n'aboutissent, comme signes visibles, qu'à la seule simplicité, comme s'il fallait l'expérience de plusieurs générations pour dégager peu à peu la courbe d'une colonne, d'une carène, ou d'un fuselage d'avion, jusqu'à leur rendre la pureté élémentaire de la courbe d'un sein ou d'une épaule.

(Saint-Exupéry 1939: 51)

Mais, dans les faits, cette tendance, quoique partout présente, ne s'exerce jamais qu'à un certain degré car les rapports entre la forme et la fonction laissent place à une certaine liberté, à un certain «jeu». Si les outils tendent vers une forme idéalement adaptée à leur fonction, de sorte que ceux qui sont destinés à une certaine fonction ont tous un air de parenté de par le monde, ils présentent aussi des différences tenant aux aléas de I'histoire et de la culture. La tendance vers l'adéquation de la forme à la fonction, tout comme l'arbitraire relatif que semblent introduire les contingences culturelles, sont l'une et l'autre sources d'une beauté, l'une liée au souci de perfection technique, l'autre recherchée pour elle-même.

En tout cela, Leroi-Gourhan ne fait au fond que reprendre la vieille question aristotélicienne du rapport entre le beau et l'utile ${ }^{\mathbf{1 1}}$, en lui apportant une réponse qui a au moins le mérite de la robustesse. Les formes d'un 


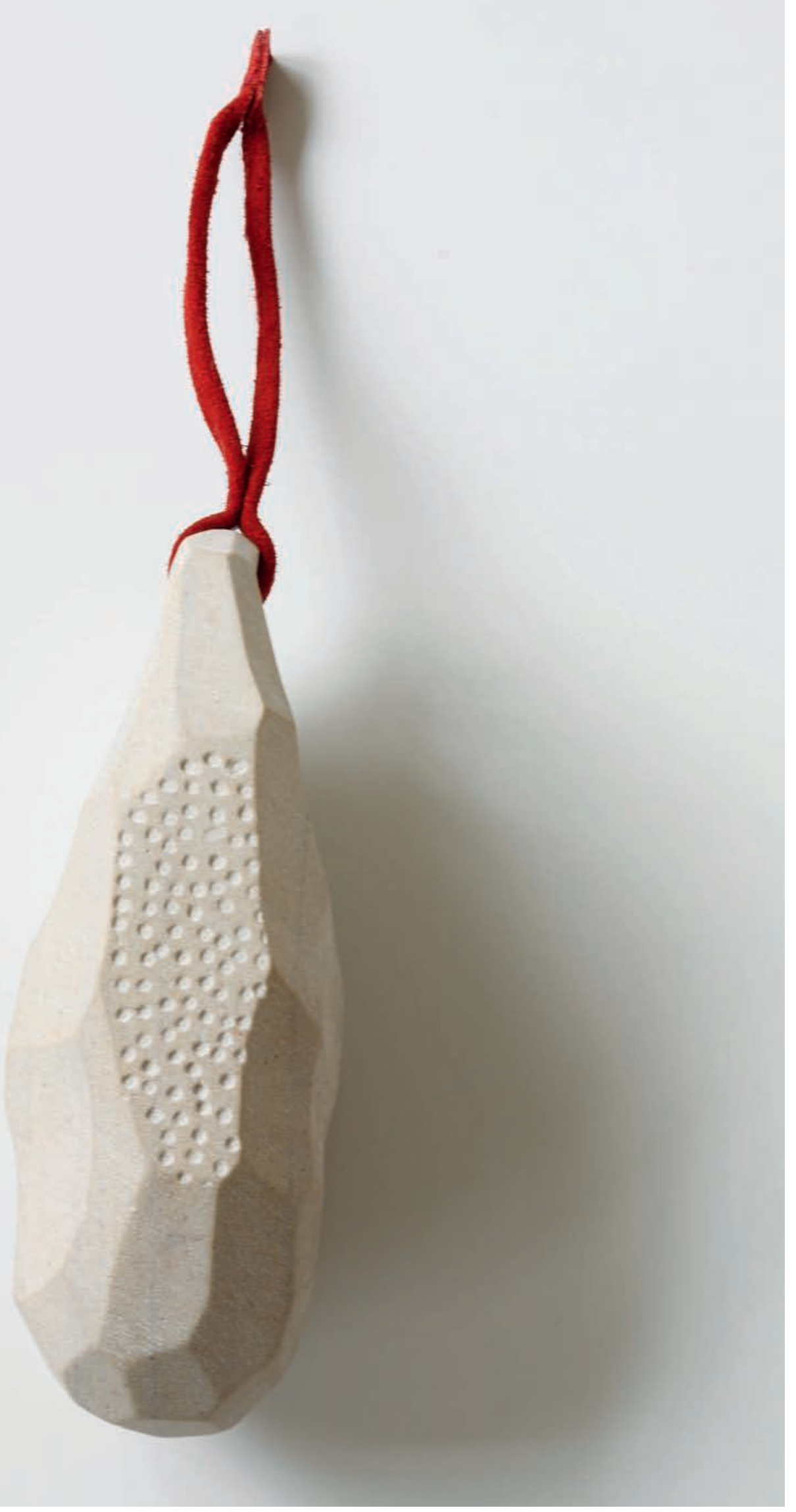


12. Les découvertes de bifaces en Europe remontent au XIX ${ }^{\ominus}$ siècle, en Afrique au début du $x x^{e}$ siècle. En revanche, les bifaces ne sont connus en Chine que depuis quelques années (voir entre autres Yamei et al. 2000). À Yunxian, au nord de la province du Hubei, les bifaces sont même encore plus anciens, autour de 936000 ans (Lumley et al. 2008). On s'aperçoit depuis peu qu'ils sont en fait largement présents en Asie.

13. Bordes 1961 ; voir aussi Camps 1979. Les bifaces les plus anciens et les plus frustes ont été qualifiés de "proto-bifaces", les plus accomplis de «bifaces évolués". On voit bien là l'effet d'une illusion rétrospective car il va de soi que tous ces bifaces, quel que soit leur degré de régularité, étaient fonctionnels et que leurs fabricants aussi bien que leurs utilisateurs n'y voyaient certainement pas des proto-outils.

14. On peut ici parler de lignée d'outils, au sens simondonien selon lequel " tout objet fait partie d'une famille ayant des ancêtres et s'inscrit dans une lignée évolutive dans laquelle l'essence technique reste stable jusqu'à la fin de la lignée »

(Deforge 1994: 174).
15. Parmi les très nombreuses études sur les bifaces, citons celle de Schick et Toth qui, au terme d'expérimentations, ont conclu qu'ils avaient souvent servi à l'équarissage du gibier à Olduvai, en Tanzanie, hypothèse confirmée par les analyses tracéologiques de Lawrence Keeley (Schick et Toth 1993).

Mais sur d'autres sites, comme à Olorgesailie, au Kenya, ils semblent avoir servi aussi de réserves d'éclats (Potts 1994).

Les auteurs d'une étude expérimentale récente visant à évaluer l'efficacité de la symétrie dans le travail de boucherie concluent que ce n'est sans doute pas le souci d'efficacité

fonctionnelle qui a incité les hominidés à rechercher la symétrie (Machin et al. 2007). Des observateurs rapportent que les aborigènes australiens utilisaient, au milieu du $x x^{e}$ siècle, les bifaces comme des pics pour ouvrir les huîtres, frapper, creuser le sol pour extraire des tubercules ou encore gratter des bâtons pour les appointer, etc. (rapporté par Brumm et Moore 2012 : 35). outil sont d'autant plus belles qu'elles sont utiles à sa fonction, et les outils fabriqués par les hommes tendraient invinciblement à évoluer vers cette beauté «fonctionnelle». Mais, dans cette séculaire évolution, ils rencontrent les aléas de l'histoire, les impondérables de la culture, dont on ne saurait dire s'ils produisent à ses yeux une beauté d'un autre ordre qui s'ajoute à la première, ou s'ils entravent la marche vers une beauté «fonctionnelle» à laquelle il attribuerait au fond une qualité supérieure.

Avant d'aller plus avant, je voudrais corriger cette approche sur un point, et je le ferai en revenant à notre biface. Il a été inventé au moins trois fois à plusieurs centaines de milliers d'années d'intervalle et dans des lieux très éloignés les uns des autres, ce qui constitue un bon exemple de «tendance» générale. II apparaît une première fois en Afrique, il y a 1,7 à 1,6 million d'années, une deuxième fois en Chine, il y a environ 800000 ans, puis une troisième fois en Europe il y a quelque 600000 ans ${ }^{\mathbf{1 2}}$. Les bifaces présentent une grande variété de formes (fig. 3), dont on a dressé le catalogue au cours des années 1960: lancéolés, amygdaloïdes, cordiformes, triangulaires, ficrons, limandes, etc., en les ordonnant généralement des plus frustes aux plus allongés et réguliers ${ }^{\mathbf{1 3}}$. Cette terminologie descriptive est encore en usage aujourd'hui. Leur variabilité morphologique n'empêche cependant pas que, dans les trois parties du monde où ils sont apparus - Afrique, Asie et Europe -, on observe la même évolution vers toujours plus de finesse et de régularité,toujours plus de rectitude dans la ligne des tranchants, toujours plus de perfection dans leur double symétrie.

L'hypothèse que l'industrie du biface ait diffusé depuis le foyer le plus ancien jusqu'aux deux autres, à la faveur de contacts ou de migrations de populations, est à exclure. Une telle diffusion aurait laissé des traces sur sa route, sous la forme de bifaces remontant aux périodes intermédiaires. Or un hiatus de plusieurs millénaires sépare les trois périodes, et les trois foyers sont séparés par des zones géographiques où l'on n'a trouvé aucune pièce bifaciale. De plus, le fait que, dans les trois cas, on repasse par les premiers stades techniques avant d'aboutir à des bifaces à la symétrie parfaite serait lui aussi difficilement explicable en cas de filiation. S'il y avait eu contact, nous ne devrions trouver que des formes évoluées parmi les plus anciens bifaces asiatiques, plus jeunes de plusieurs centaines de milliers d'années que les bifaces africains. La documentation archéologique semble donc attester que les fabricants de bifaces auraient été poussés à adopter la forme la plus simple et, à nos yeux du moins, la plus belle, que nous voyons réalisée dans les bifaces les plus tardifs ${ }^{\mathbf{1 4}}$.

Cette évolution est-elle simplement due, comme le pensait LeroiGourhan, à la recherche obstinée de la meilleure adéquation entre la forme de l'outil et sa fonction? On en est moins sûr aujourd'hui, pour une raison très simple: l'observation des tranchants au microscope électronique à balayage et des expérimentations pour certaines déjà anciennes permettent de conclure qu'il s'agissait vraisemblablement d'outils à tout faire (de couteaux suisses!) dont on utilisait aussi bien les tranchants que la pointe et avec lesquels on pouvait couper, gratter, piquer... II est en tout cas certain qu'il ne s'agissait pas de pointes de jet comme on l'a parfois cru, car ils auraient été beaucoup trop lourds et volumineux pour un tel usage ${ }^{15}$. On n'est donc 
plus tout à fait certain que la symétrie ait joué un rôle fonctionnel décisif: difficile de parler d'adéquation de la forme à la fonction quand l'outil a servi à tout et n'importe quoi ${ }^{\mathbf{1 6}}$ (fig. 6).

Mais quelque chose d'autre a pu jouer dans cette évolution vers une forme de plus en plus symétrique. Regardons à nouveau notre biface. II se résume à la rencontre de deux tranchants qui se rejoignent de manière à former une pointe. Or, chacun de ces tranchants est lui-même constitué par l'intersection de deux plans sécants. Pour l'artisan qui fabrique le biface, le problème se ramène donc, si l'on s'en tient à la partie active de l'outil, à réaliser quatre plans formant un tétraèdre dont deux arêtes sont très aiguës, et deux autres sont au contraire émoussées, ce qui donne une section en forme de losange aplati. Il obtient ce résultat par approximations successives en procédant à des retouches par pression sur un plan, puis sur l'autre, etc., et le travail qui s'exerce sur chacun des plans est le même: il est réalisé petit à petit au prix d'un effort assez prolongé (qui peut aller jusqu'à une heure pour un expérimentateur moderne ${ }^{\mathbf{1 7}}$ ), de sorte que, assez naturellement, la fabrication de l'outil pèse en faveur d'un objet disposant de deux plans de symétrie. Tout simplement parce qu'on a travaillé de la même manière sur les quatre faces. Imaginons en effet l'ouvrier qui contemple l'une des faces de son tétraèdre et juge que telle retouche permettra d'améliorer la rectitude de son arête tranchante; il effectue donc cette retouche, puis retourne son biface et examine semblablement une autre face, la retouche à son tour, etc. À chaque retouche, sur chaque face, son souci reste le même: rendre l'arête tranchante la plus rectiligne et la plus aiguë possible. Les gestes qu'il fera à chacune de ses frappes, dont un long apprentissage lui a appris à doser l'angle et la force, se ressembleront tous. Et les quatre faces qu'il produira ainsi peu à peu se ressembleront, si bien que, sans l'avoir recherché mais simplement parce que ses gestes seront toujours semblables, l'outil finira par avoir deux plans de symétrie. La forme si dépouillée que le biface a fini par atteindre, cette économie géométrique qui portait Leroi-Gourhan à lui attribuer une beauté «fonctionnelle», serait donc peut-être due aussi au fait que les artisans qui les ont fabriqués ont su se montrer de plus en plus économes. Non qu'ils auraient été de plus en plus avares de leurs gestes car il semble au contraire que le nombre de mouvements exécutés s'est accru à mesure que l'outil a évolué. Mais ils ont laissé disparaître les gestes parasites, et s'en sont tenus avec de plus en plus de rigueur seulement à ceux exigés par la fin à atteindre, qu'ils ont aussi appris à adapter à la matière première utilisée. Cette économie du geste - qui doit être direct et non «besogneux» - est aujourd'hui encore un critère de qualité d'exécution chez les artisans, comme le remarque Baptiste Buob dans ce volume à partir de ses observations de la pratique des luthiers.

Un artisan expérimenté qui a déjà fabriqué de nombreux bifaces et sait donc ce que le processus de fabrication est naturellement porté à produire a en tête cette symétrie. Sur ce point, je rejoins tout à fait l'opinion de Jacques Pelegrin. Vieux d'environ 700000 ans, les bifaces exhumés par centaines sur le site d'Isenya, au Kenya, qui répètent la même forme régulière en amande, témoignent selon lui que leurs auteurs avaient en tête un modèle, une image mentale de cette forme, et que les nombreux enlèvements de façonnage ont été ajustés, selon un agencement et un ordre
16. Sur la question de l'éternel problème de la définition juste de la fonction et de la difficulté de déduire une fonction d'une forme, voir Graves-Brown

2000 et Preston 2000 Parmi les nombreux cas que l'on peut évoquer. ceux des cuillères et des mortiers qui servent d'instruments de musique sont les plus souvent cités. Le cas des timbales en terre cuite des Itcha du Bénin, qui ne sont plus fabriquées aujourd'hui que pour servir d'instruments de musique alors qu'elles étaient primitivement destinées aux deux fonctions, est particulièrement parlant (Madeleine Leclair, "Musique et changement social chez les Itcha du Bénin ", exposé dans le séminaire "Identités en scènes, cultures en actes " organisé par Thierry Bonnot et Bernard Müller, musée du quai Branly, 10 mars 2005). Reste à savoir si la symétrie de leurs formes correspondait à leur usage alimentaire ou aux nécessités de la sonorité. J'ai moi-même observé chez les Touaregs des mortiers utilisés comme instruments de musique en position retournée et recouverts d'une peau, le temps d'une soirée, mais qui reprenaient leur fonction d'ustensile culinaire dès le lendemain.

Il est évident dans ce cas que la forme (et donc la symétrie) de l'objet est d'abord dictée par les impératifs de son usage comme mortier. La preuve en est qu'ils ont exactement la même forme que les mortiers utilisés par les populations voisines, lesquelles ne les transforment jamais en instruments de musique (Dominique Casajus, communication orale).
17. Frédéric Abbès, de la Maison de l'Orient méditerranéen, vient régulièrement faire des démonstrations de taille de silex devant mes étudiants et je puis donc témoigner que, malgré sa dextérité et son expérience, il ne lui faut pas moins d'une demiheure à une heure pour obtenir un biface. 


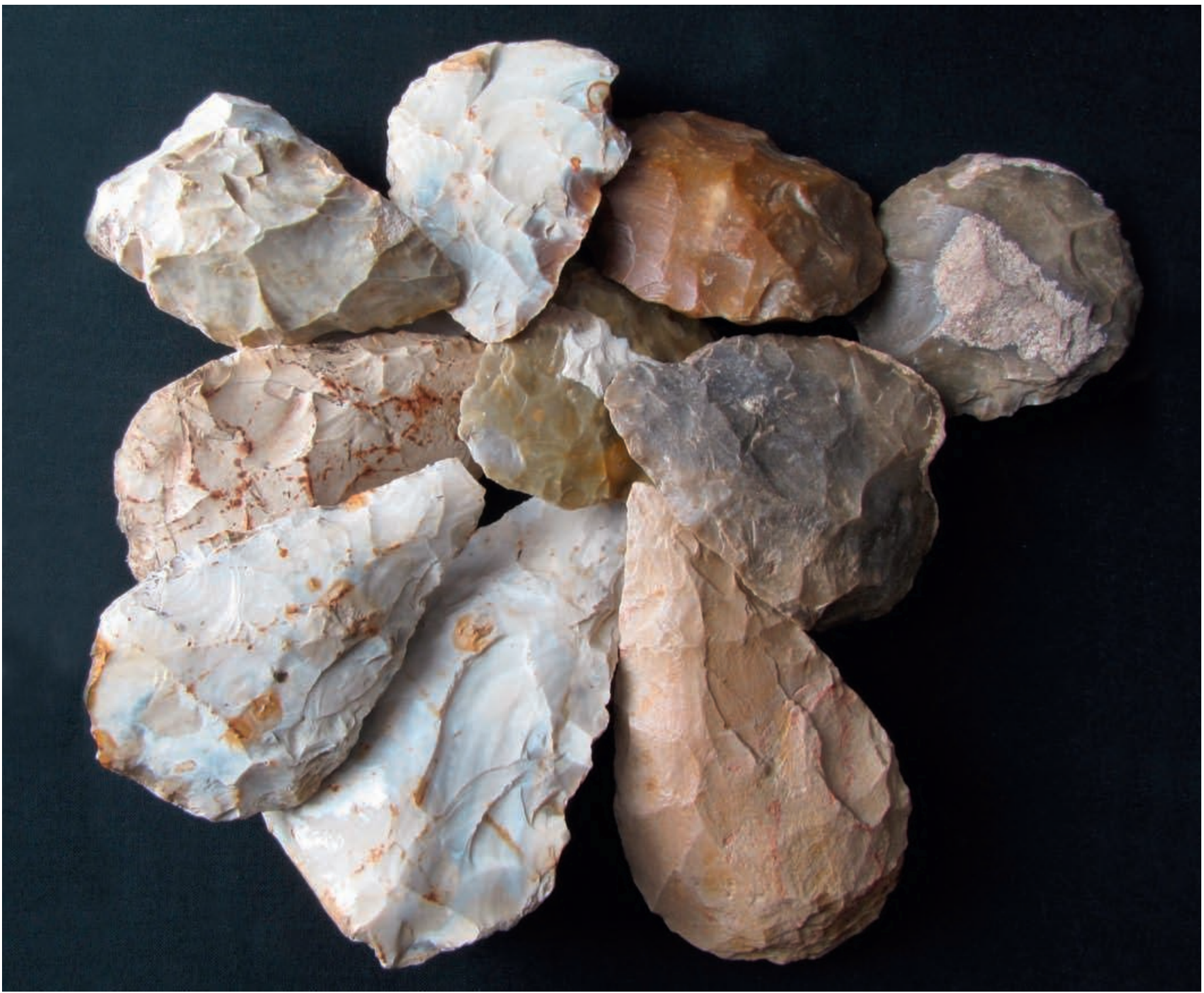

fig. 3

Série de bifaces des vallées de la Somme, de l'Oise et de l'Aisne découverte fin $x x^{\ominus}$ - début $x x^{e}$, Paléolithique inférieur et moyen (300000 à 40000 ans av. J.-C.). (c) Jean Luc Katzman (www.aggsbach.de) variables, de façon à reproduire cette forme préconçue ${ }^{\mathbf{1 8}}$. Notre fabricant de biface procède ainsi probablement parce que tous les bifaces qu'il a déjà fabriqués, hormis peut-être les premiers dont la forme était encore imprécise, lui ont peu à peu dicté cette exigence de symétrie. II ne la recherche peutêtre pas, mais il sait d'expérience qu'elle finira par apparaître, et la laisse donc naître peu à peu de ses enlèvements et retouches successifs. II est probable que cette symétrie à laquelle nous sommes aujourd'hui si sensibles lui paraissait belle à lui aussi. En tout cas, s'affirmant au fur et à mesure que ses retouches se succédaient, elle ne pouvait que réjouir son cœur et son esprit car elle était le signe qu'il faisait de la belle ouvrage, que l'outil qui naissait peu à peu de ses mains serait un bon outil. II éprouvait de la joie à la faire naître car il n'y a pas de raison de lui refuser ce plaisir de faire, partagé par tous les artisans, qui est pour François Sigaut un puissant moteur de la créativité humaine, voire ce qui expliquerait que l'action outillée soit une exception chez l'animal et la règle chez l'homme ${ }^{19}$. II éprouvait aussi 
de la joie à la contempler. Cette joie de l'âme à la contemplation d'une forme satisfaisante, qu'est-elle donc sinon le sentiment du beau? C'était en tout cas l'avis de Marcel Mauss, pour qui «l'objet esthétique est un objet que l'on peut contempler, il y a dans le fait esthétique un élément de contemplation, de satisfaction en dehors du besoin immédiat, une joie sensuelle mais désintéressée ${ }^{20}$ ».

Si le lecteur a accepté de me suivre jusqu'ici, il m'accordera donc que les anciens représentants du genre Homo à qui nous devons les bifaces étaient aptes à ce sentiment. Que Homo sapiens ait été sensible au beau, des peintures pariétales ou des statuettes vieilles de plusieurs dizaines de milliers d'années nous en ont depuis longtemps apporté la preuve. Les êtres plus humbles mais déjà humains qui l'ont précédé sur terre ne lui étaient pas, de ce point de vue, inférieurs. Le souci du beau serait donc plus ancien que notre espèce. Mais peut-être pas plus ancien que le genre Homo: les primates non humains sont incapables de créer des outils symétriques. Bien sûr, le sentiment du beau ne se réduit pas au souci de la symétrie. Simplement, ma longue méditation sur le biface m'a donné la conviction que la symétrie de ses formes était pour ceux qui le fabriquaient la source d'une émotion qu'il me faut bien qualifier d'esthétique ${ }^{\mathbf{2 1}}$. Peut-être les primates sont-ils susceptibles d'éprouver des émotions de ce genre, mais on voit mal comment on pourrait le prouver. Notons par ailleurs qu'ils sont également incapables de produire des battements rythmés: la capacité à garder le tempo serait spécifiquement humaine (Gärdenfors 2007 [2004]: 226-227). Leroi-Gourhan a lui aussi souligné que «l'une des caractéristiques opératoires de l'humanité, dès ses premiers stades, a été l'application de percussions rythmiques, longtemps répétées ${ }^{22}$ ». Nous voilà loin du biface? Pas sûr. La double incapacité que nous découvrons ici au primate pourrait bien n'en faire qu'une puisque le rythme a été décrit comme la symétrie du temps (ibid. : 227). Et Mauss, toujours lui, ne disait-il pas, à la suite de Franz Boas, que si la notion de beau peut être associée à la notion de plaisir et de joie, il faut aussi la rattacher au rythme, «car là où il y a rythme, généralement il y a esthétique» (Mauss 1967 [1947]: 87; Boas 2003 [1927]).

Mais revenons aux outils. L'idée d'une tendance qui pousse les outils vers une certaine forme est sans doute à garder; et il n'y a pas lieu de nier qu'elle correspond à la recherche d'une meilleure efficacité technique. II faut simplement ajouter une deuxième idée: cette tendance correspond aussi à la recherche d'une meilleure économie gestuelle dans la fabrication de l'outil. Retenons donc la notion leroi-gourhanienne de tendance, mais en prenant garde que cette tendance est double. Et on peut aussi garder l'idée que les aléas de la culture ou de l'histoire sont susceptibles, dans les faits, de faire dévier cette double tendance. Et ce, même dans le cas d'un objet aussi simple que le biface. C'est ce qui s'est passé pour le biface acheuléen provenant de West Tofts en Angleterre (fig. 4). Il a été taillé en tenant compte de la présence, dans le bloc de matière première, d'un coquillage fossile. Le tailleur s'est arrangé pour façonner son biface de manière que le coquillage apparaisse au centre d'une des faces, comme s'il fallait mieux mettre en valeur la symétrie de l'objet. Il est évident ici qu'il s'agit d'un élément déco-
18. Pelegrin 2009. François Sigaut va encore plus loin en évoquant la nécessité d'un «modèle mental de l'action outillée" (Sigaut 2012: 97).

19. Sigaut 2012. Le plaisir de faire de la belle ouvrage peut même se révéler dans des détails non visibles mais connus seulement de l'artisan ou de l'artiste. Il en est ainsi des grands bronzes de l'Antiquité, dont les parois sont de plus en plus fines et régulières au fil des siècles - 7 à 9 mm en moyenne avant le $v^{\mathrm{e}}$ siècle av. J.-C. pour atteindre 2 à $5 \mathrm{~mm}$ à la période romaine. Une telle finesse rend la coulée très difficile à réussir et sa réalisation suppose d'une part une connaissance approfondie des procédés de moulage des plus grands sculpteurs-fondeurs de la Grèce archaïque et classique, d'autre part un très grand savoir-faire (Mille et Darblade 2012). Ce qui s'apparente à une véritable prouesse technique évoque un défi d'artisan.

20. Mauss 1967 [1947] : 86. Alfred Gell parle pour sa part de l'enchantement lié à la fascination exercée par l'excellence technique (Gell 1998). En établissant un lien entre la virtuosité technique et le pouvoir de fascination de l'œuvre, il réduit l'esthétique au désintéressement et ne conçoit pas que le beau soit efficace, ce qui n'est évidemment pas notre cas ici.
21. Lors d'un échange constructif avec Thierry Bonnot, celui-ci me faisait remarquer que l'on peut s'interroger sur le type de plaisir particulier éprouvé par le tourneur potier payé à la pièce qui travaillait dans les usines de poteries en grès après la réussite d'un cruchon aux formes particulièrement harmonieuses: satisfaction d'avoir répondu au modèle formel imposé par la direction de l'usine, émotion esthétique ou un peu des deux à la fois? On peut aussi se faire l'" avocat du diable" en évoquant le cas de certains collectionneurs à la recherche de l'objet dysharmonique qui présente un défaut de fabrication, ce qui en fait un objet unique peut-être plus émouvant car portant la trace du geste et de l'outil. Mais c'est au fond une sorte de perversion d'esthète qui se construit par opposition à une «norme » esthétique.

22. Leroi-Gourhan 1965 : 135. Les italiques sont de nous. Alexandra Bidet a consacré un bel article au thème de la rythmicité qui traverse l'œuvre d'André Leroi-Gourhan (Bidet 2007). 


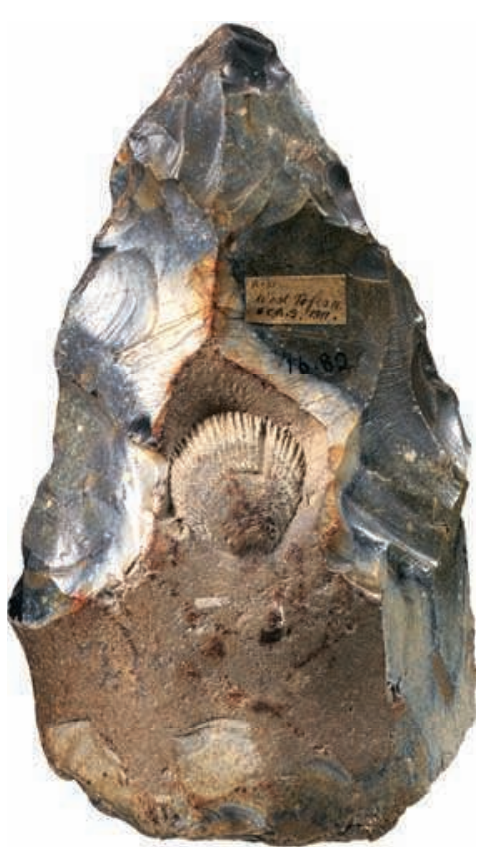

ci-dessus

fig. 4

Biface de West Tofts, Norfolk (Royaume-Uni), fin de l'Acheuléen ou Palélothique moyen, vers 100000 av. J.-C. Cambridge, Museum of Archaeology and Anthropology.

\section{ci-contre}

fig. 5

Cuillère pyrogravée en bois d'acacia d'origine touarègue, Mali, groupe des loullemmeden Kel Ataram, $x x^{e}$ siècle. (C) 2013 Genève musée d'Ethnographie. ratif qui ne présente aucune utilité pratique. II atteste peut-être aussi que, même si la symétrie tenait à la destination de l'outil et à sa fabrication, elle a peut-être fini par être recherchée pour elle-même. Le fait ne vient pas ici perturber la tendance, mais la magnifier.

Mes propositions ont, j'en conviens, quelque chose d'hypothétique, ne serait-ce que parce que nous n'avons pas été témoins de la fabrication de bifaces autres qu'expérimentaux. Je vais donc parler d'un autre objet, tout aussi simple, mais sur la fabrication duquel j'ai pu faire des observations: une cuillère touarègue en bois taillée dans la masse (fig. 5). La courbure de son dos prolonge sans rupture notable celle de son manche, lui donnant à mes yeux une beauté à laquelle les Touaregs sont certainement sensibles eux aussi. Elle est par ailleurs ornée de dessins pyrogravés, qu'ils doivent aussi juger beaux, mais qui n'ajoutent rien à sa fonction au sens que la cuillère resterait une cuillère même en leur absence: ils sont l'équivalent du coquillage du biface de West Tofts. Sans doute resterait-elle une cuillère même si la courbe de son manche faisait un angle avec celle de son dos, mais l'adaptation de la forme à la fonction serait alors moins parfaite, moins économe. Est-ce cette économie que les artisans touaregs ont recherchée? Si c'est le cas, alors nous pouvons les considérer comme de parfaits designers. Mais peut-être se sont-ils également souciés d'être économes de leurs gestes quand il l'ont fabriquée, à l'instar des luthiers observés par Baptiste Buob. On peut penser en effet - mais des observations précises permettraient seules d'être affirmatif - que s'est peu à peu imposée une forme facile à fabriquer, une forme qui se laisse sculpter plus aisément, avec une moins grande variété de gestes. Si la courbure du manche faisait un angle avec celle du dos de la cuillère, l'artisan qui taille l'objet devrait modifier l'orientation de son herminette au moment où la progression de son travail le fait passer d'une courbure à l'autre. Alors que cette courbure s'obtient par une série de petits coups d'herminette imprimés à la cuillère pendant que l'artisan la fait se mouvoir dans sa main gauche par une rotation continue de son poignet gauche, tandis qu'il tient l'outil, toujours orienté de la même façon, avec sa main droite.

Récapitulons nos acquis. La forme de ma cuillère touarègue s'adapte avec économie à sa fonction. Sa beauté vient de ce que ses formes sont minimales: aucune superfluité de forme ne s'ajoute à ce qui est nécessaire pour qu'elle soit une bonne cuillère (alors que si la courbure de son manche ne prolongeait pas parfaitement la courbure de sa partie convexe, il y aurait entre l'une et l'autre un angle, une discontinuité «superflue»). Et sans doute devait-il en être de même pour le biface, même si l'ignorance où nous sommes de sa fonction nous interdit d'être aussi affirmatifs. Cette économie est source d'une beauté que Leroi-Gourhan qualifiait de «fonctionnelle». Ici, le beau, c'est l'utile, mais un utile ascétiquement réduit à lui-même. Par ailleurs, ces formes économes sont le fruit d'un travail lui-même économe, d'une gestuelle tout aussi ascétique. II est permis d'admirer que, pour nos deux objets, et sans doute aussi pour beaucoup d'objets fabriqués à la main, la fabrication la plus économe soit précisément celle qui permet de produire la forme la plus économiquement, la plus purement, adaptée à la fonction. Cette harmonie ne s'impose pas de façon aussi naturelle lorsque le concepteur est distinct du fabricant, comme c'est le cas pour 


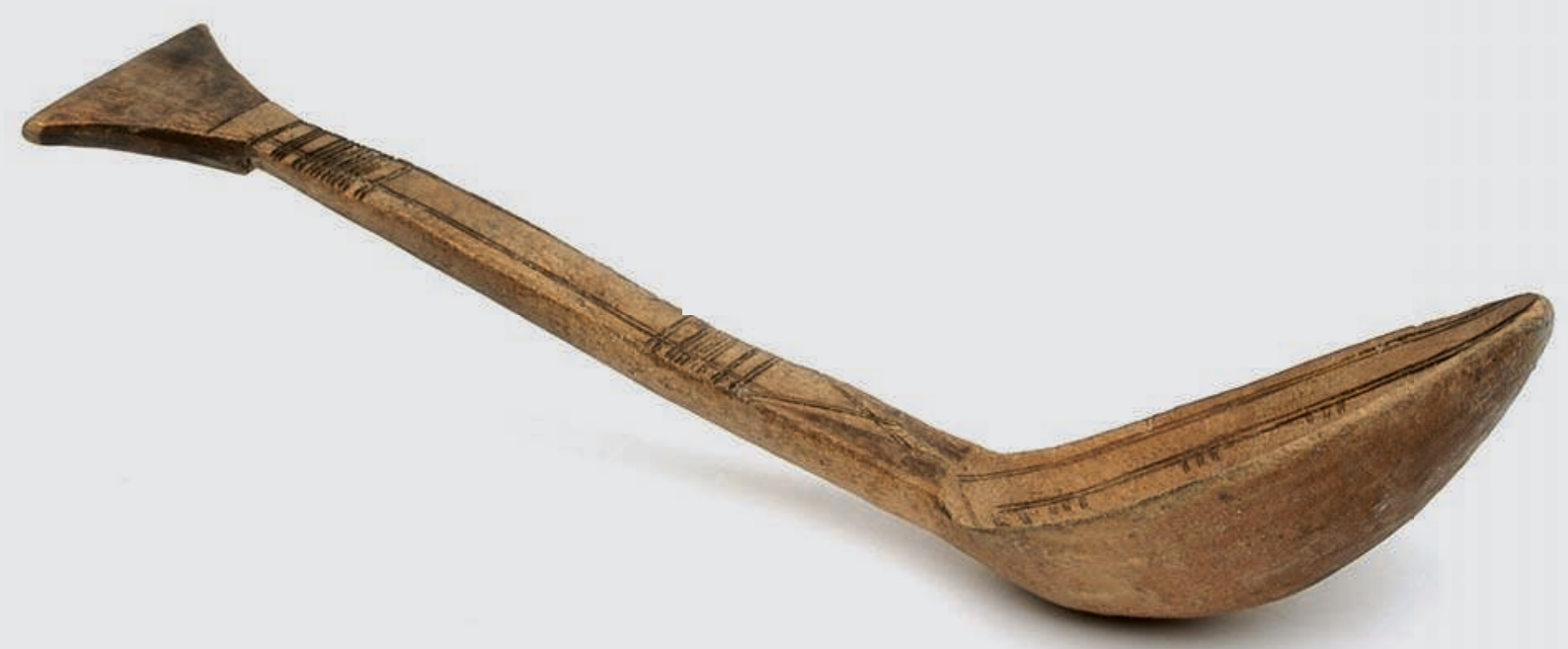




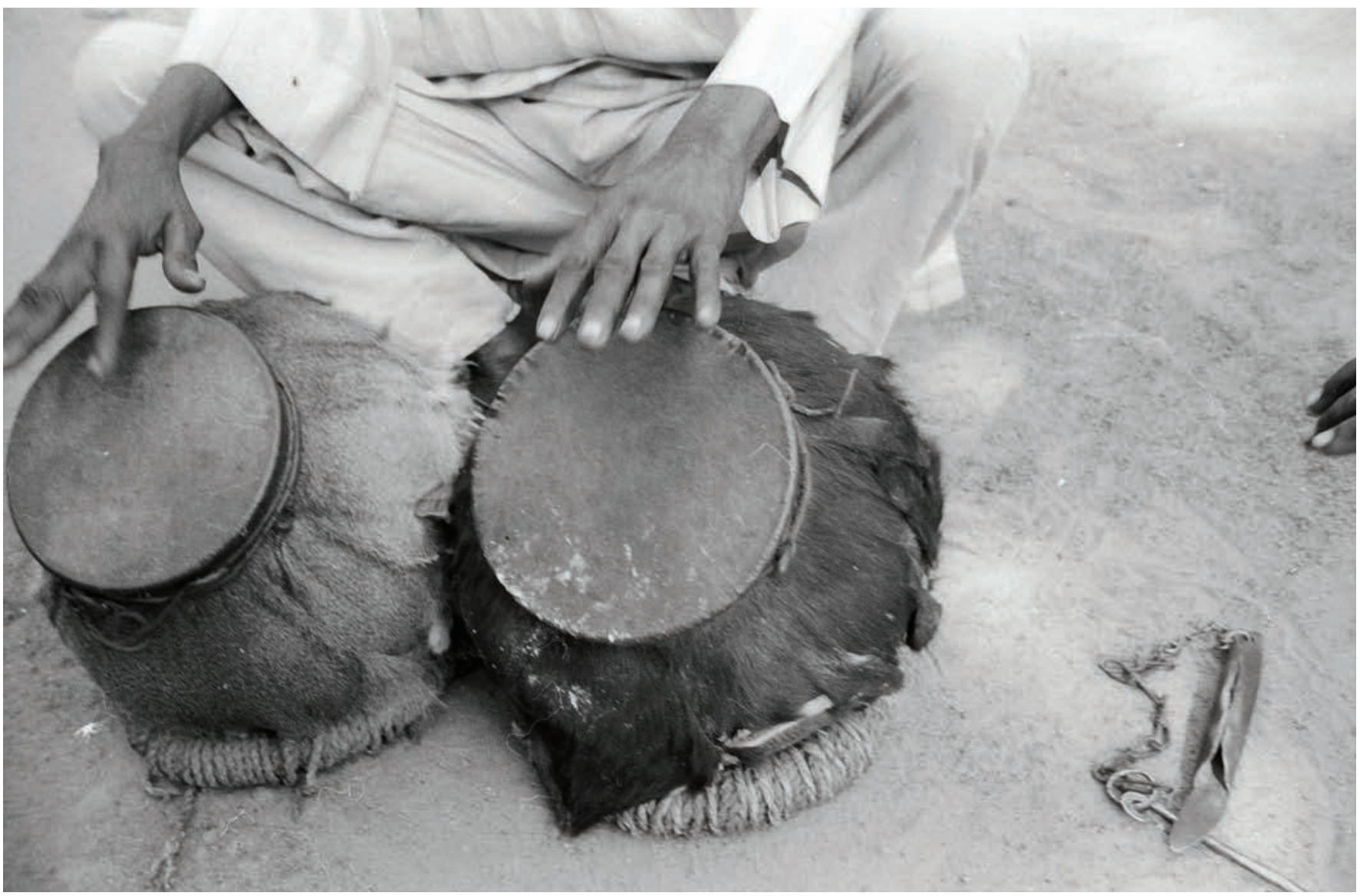

fig. 6

Timbales kolobi composées de jarres en poterie recouverte de peau, Bénin, village de Pira, population itcha (sous-groupe yoruba), 1998. (C) Madeleine Leclair. les designers actuels. C'est dire que, si la recherche de la beauté fonctionnelle telle que la définissait Leroi-Gourhan rapproche les artisans préhistoriques (ou les artisans touaregs) de nos modernes designers, le rapprochement se justifie moins si l'on doit admettre que la beauté des outils tient aussi à l'économie des gestes qui les ont produits. En effet, il est peu probable qu'un designer se soucie de la gestuelle de l'artisan qui devra fabriquer les outils qu'il conçoit puisqu'il s'agira d'objets fabriqués en série, et par des machines.

Rien n'interdit de trouver de la beauté aux gestes simples et économes du fabricant d'outils (comme on le sait, il y a des gestes que leur simplicité rend augustes). De cette beauté, Leroi-Gourhan n'a rien dit, alors qu'elle est au fond de même nature que sa beauté «fonctionnelle» puisque les beaux gestes du fabricant sont parfaitement adaptés à leur destination. C'est que la fabrication des outils l'a moins intéressé que leur utilisation. Et même pour ce qui est des gestes de l'utilisateur, il en est resté à une typologie assez générale (couper, frapper, etc. ${ }^{23}$ ). II n'en a pas été de même pour André-Georges Haudricourt, qui ne concevait pas l'outil sans prendre en compte les forces motrices qui le font agir, et en particulier le moteur humain. D'un côté donc, une approche qui privilégie l'outil et son action sur la matière, de l'autre une vision englobant l'outil et la force qui le meut. Haudricourt s'est aussi intéressé au fabricant, et a souligné combien «on est toujours impressionné 
par l'objet concret, qu'il soit outil ou objet fabriqué, et l'on oublie de penser au bras humain - la force qui fait mouvoir cet outil - et au travail dépensé pour fabriquer un objet» (Haudricourt 2010: 26). C'est de ce travail que j'ai parlé ici, et je pense avoir montré qu'il peut être source de beauté.

\section{Fonction identique, formes et décors variables}

Nous n'en avons pas encore fini avec notre biface. L'importance que j'accorde aux gestes nécessaires à sa fabrication permet, me semble-t-il, de rendre compte du fait que, dans chacune des trois régions où ils sont apparus, les bifaces ont évolué vers une double symétrie de plus en plus parfaite. Les bifaces recueillis par les archéologues ont cependant des formes variables, certes de plus en plus symétriques au fur et à mesure que le temps passe, mais variables tout de même. Et il ne faut pas espérer s'en tirer en disant que c'est parce qu'ils avaient des fonctions variables, étant donné que, comme on l'a vu, les exemples examinés d'assez près semblent avoir servi à toutes sortes d'usages. II faut donc affiner nos remarques précédentes. Des gestes toujours semblables à eux-mêmes tendent à doter le biface d'un double plan de symétrie. Mais si ces gestes restent les mêmes pour un artisan donné, ils peuvent varier un peu d'un artisan à l'autre, et varier davantage encore d'une région à l'autre. Chacun les a appris en regardant faire son maître, et les a reproduits avec son tempérament personnel qui ne sera pas exactement le même que celui de ses condisciples. D'où des variations individuelles, peu perceptibles peut-être en un lieu donné, mais qui, dans des régions différentes, définissent des «écoles», des styles, qui «s'insinu[ent] dans la marge étroite que la fonction laisse disponible à la forme 24 ».

Les variantes régionales peuvent évoluer avec le temps, mais cette évolution ne se laisse voir que sur le très long terme. On sait en effet que les mouvements corporels sont une caractéristique culturelle stable et résistante au changement, et ne se modifient que très lentement. Pensons à l'exemple de l'écriture, qui du Moyen Âge jusqu'au XIX ${ }^{e}$ siècle se pratiqua à main levée (David 1997: 95). Et les gestes des fabricants résistent d'autant plus aux changements à court terme que les artisans d'une région donnée peuvent être attachés aux formes singulières qu'ils ont l'habitude de produire, surtout s'ils savent qu'elles diffèrent un peu de celles que produisent leurs voisins. Les ethnographes ont recueilli des exemples qui vont bien dans ce sens, en les interprétant toutefois avec, me semble-t-il, un peu trop de raideur. Ainsi lan Hodder croit pouvoir affirmer que les données qu'il a recueillies au Kenya et en Zambie montrent que la distribution des types différents d'un même outil, surtout dans les zones frontalières où la tension et la compétition sont les plus vives, résulterait d'une volonté de renforcer l'identité des groupes (Hodder 1982). De même, Pierre Lemonnier pense que chaque groupe tend à faire varier ses outillages et ses techniques pour se démarquer de ses voisins, en n'hésitant pas pour ce faire «à se mettre à dos le déterminisme de la nature $\mathbf{2 5}$ ». Pour Marie-Claude Mahias, la cohésion et la rigidité des groupes sociaux est telle que «tout changement, dans les outils comme dans le régime alimentaire, risque de remettre en cause l'ordre social et l'adoption d'un trait nouveau engendre simultanément un système technique et une entité sociale» (Mahias 1994: 193). Leroi-Gourhan évoquait d'ailleurs la notion de «style ethnique» à propos
23. II reconnaissait toutefois que les gestes pouvaient revêtir des styles différents et que ces nuances dans l'utilisation étaient peut-être au fond le seul élément réellement significatif. II notait non sans humour que, si l'on peut dresser une carte

de la répartition des populations qui mangent avec des baguettes, il est quasi impossible de restituer celle de ceux qui font mouvoir leurs baguettes «à la japonaise, à la chinoise, à la mongole, avec vulgarité ou avec raffinement » (Leroi-Gourhan 1968: 1823).

24. Leroi-Gourhan 1965 : 132. Le style ne se limite pas à l'esthétique puisqu'il peut traduire différentes manières de faire la même chose. Ce sont les fameuses variations "isochrestiques " - à usage égal - de James Sackett (Sackett 1990). Les variations stylistiques et leur signification ont donné lieu à de riches débats dans les années 1980-1990. Les reprendre ici déborderait le cadre de cet article. Pour une revue récente de la question, voir Martinelli 2005.
25. Lemonnier 1991: 19, et surtout 1993. Ces choix dictés par des raisons plus sociales que techniques constituent le thème d'un ouvrage de Marshall Sahlins dans lequel celui-ci insiste sur le fait que, si les contraintes techniques fixent des limites, la marge laissée au groupe à l'intérieur de ces limites est assez grande pour qu'il puisse poser des choix d'ordre culturel indépendamment de - ou antérieurement à ses choix techniques (Sahlins 1980 [1976]) 


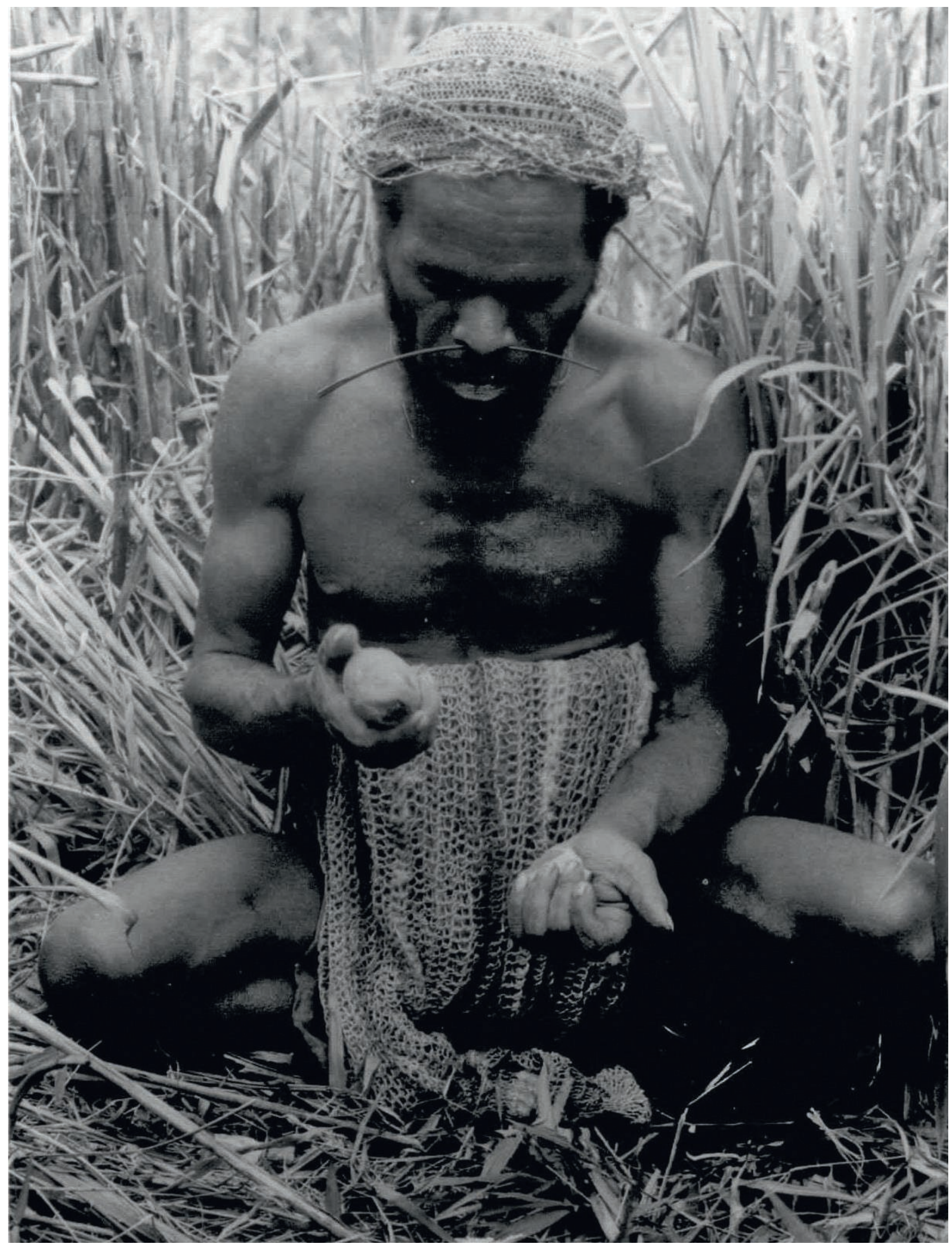


d'objets très contemporains: «Les voitures de course anglaises, italiennes et américaines sont en état d'approximation fonctionnelle puisqu'elles conservent un style ethnique malgré les exigences de l'aérodynamisme qui devraient les faire identiques. "(Leroi-Gourhan 1965: 130) On peut rapprocher le «style ethnique» de Leroi-Gourhan du «tissu social» de Paul Zumthor, une cohérence esthétique et iconique en laquelle les hommes se reconnaissent (Zumthor 1993, cité par Coquet 2012). Mais aller jusqu'à parler de souci de «cohésion sociale», imaginer que la modification d'un outil puisse la remettre en cause, c'est attribuer à l'ensemble des traits d'une société plus d'interdépendance qu'ils n'en ont. De plus, les manières de faire n'ont pas toujours forcément quelque chose à voir avec un sentiment d'identité ${ }^{26}$. Comme le fait justement remarquer Bruno Martinelli, certains traits matériels "peuvent être mobilisés pour des assignations statutaires, des qualifications entre groupes et catégories au sein d'une même société, comme entre sociétés ou ethnies", et d'autres pas. Et ces traits - faits vestimentaires, capillaires, culinaires ou architecturaux - peuvent d'ailleurs passer d'un registre à l'autre $\mathbf{2 7}^{\mathbf{2 7}}$.

II est plus simple de penser que les fabricants sont attachés à la forme des outils qu'ils savent façonner tout simplement parce que celleci est le fruit d'un long et difficile apprentissage qui remonte à l'enfance. Cet attachement fait qu'ils considèrent que c'est la seule vraie, la seule bonne, et que leurs voisins, attachés pour les mêmes raisons à une forme légèrement différente, sont dans l'erreur et «ne savent pas ce qui est beau ». Gilbert Simondon ne disait pas autre chose quand il remarquait que l'acquisition précoce des connaissances techniques par imprégnation quotidienne profonde dès le plus jeune âge tend à empêcher de repenser, rediscuter et renégocier ces connaissances à l'âge adulte (Simondon 1958: 89). Il suffit de regarder autour de nous, et même en nous-mêmes, pour percevoir qu'il n'y a rien là de bien exotique. Mais ici aussi, il faut sans doute nuancer, comme le fait Olivier Gosselain qui distingue, à propos des potiers du Cameroun, d'une part les compétences techniques difficiles à assimiler, qui sont les plus fortement enracinées car apprises très jeunes - il parle à leur sujet d'«enracinement moteur» à la suite de Dean Arnold - et sont effectivement stables et très résistantes aux changements, et d'autre part les compétences acquises par la suite et susceptibles de modifications (Arnold 1981; Gosselain 2012; Simondon 1958). Du reste, si les savoir-faire étaient aussi rigides, on ne pourrait même pas imaginer qu'ils puissent évoluer pour déboucher sur des innovations. Or il est évident que les contacts entre populations voisines ont engendré emprunts, échanges et circulation d'idées, de savoir-faire mais aussi d'objets, et ce quel que soit le contexte historique, ethnologique ou archéologique ${ }^{28}$.

J'ai raisonné pour l'essentiel à partir d'un objet de forme simple: un biface, si je m'en tiens à sa partie active, n'est jamais qu'un tétraèdre. L'épure que j'ai ainsi esquissée se brouillerait sans doute si l'on devait considérer des objets aux formes moins faciles à circonscrire. Ainsi, que penser de l'extrême variété des formes de pointes de flèches provenant du Néolithique saharien, de l'Amérique précolombienne ou du Mésolithique européen: variation du rapport entre la longueur et la largeur, de l'ouverture de l'angle du corps, subtiles différences de convexité ou de concavité des
26. Sur les questions d'identité et de la part qu'y prend la culture matérielle, voir en particulier Lemonnier (éd.) 1993, et surtout l'introduction au volume.

27. Martinelli $2005: 27$. Peut-être faut-il, comme le préconise Christian Bromberger, distinguer les signes socialement pertinents, les marqueurs, des faits de style inconscients qui s'expriment dans les habitus (Bromberger 1973).

28. Voir sur ce point Beaune 2008, mais aussi, pour un exemple historique, Hilaire-Pérez 2000, à propos des modalités de transferts technologiques au Siècle des lumières.

\section{ci-contre}

fig. 7

Mayka Kot (groupe des Wola du Sud des Hautes Terres) taillant une pointe de flèche en silex, Nouvelle-Guinée, 1978, avec l'aimable autorisation de Karen Hardy et Paul Sillitoe (in Internet Archaeology 14). (c) Photo Paul Sillitoe. 


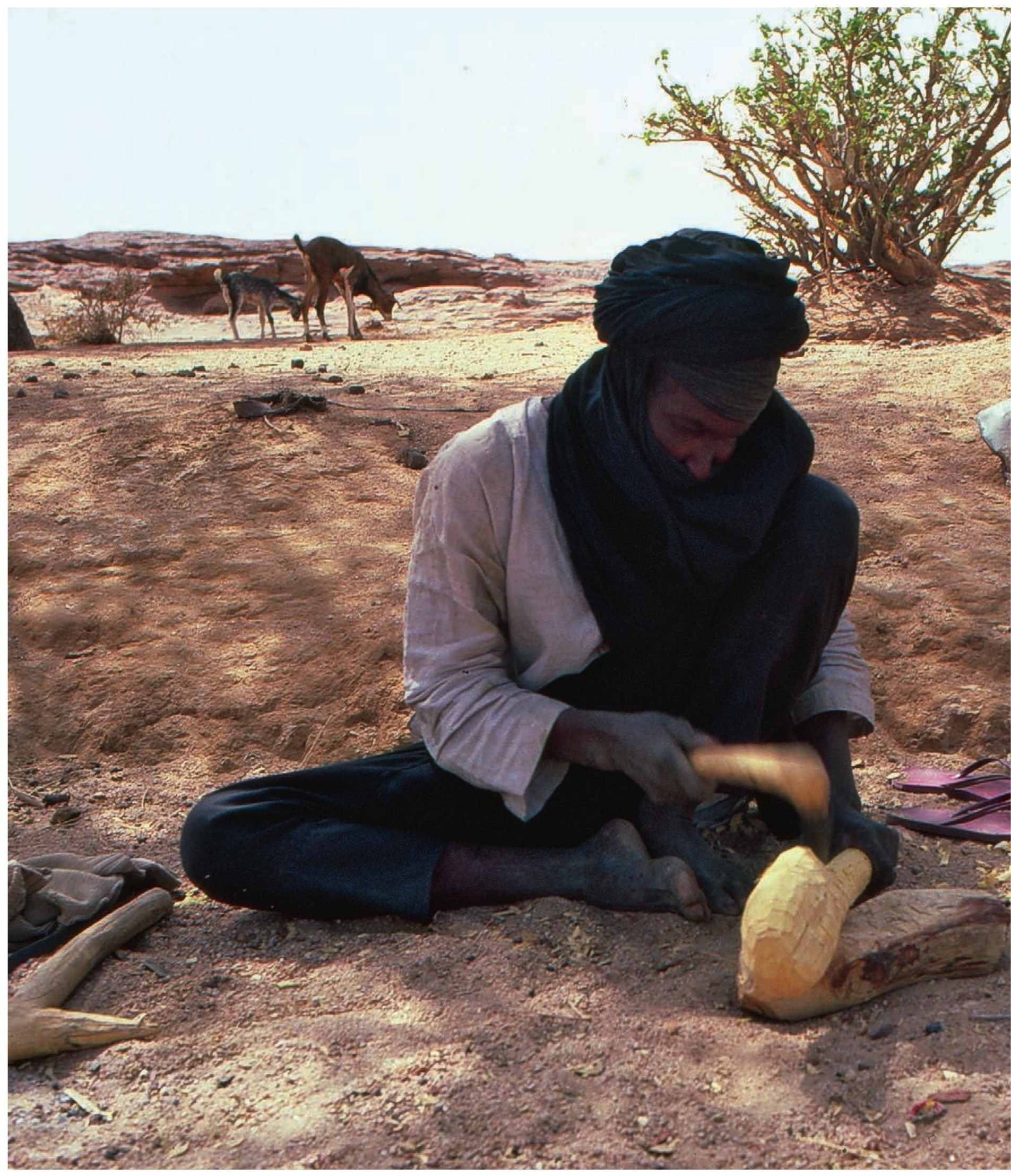


29. Voir par exemple Marchand 2000 pour diverses hypothèses pouvant expliquer la variabilité des objets techniques au Mésolithique; Camps 1979, pour des exemples de typologies de pointes de flèches mésolithiques et néolithiques. tranchants, fluctuation de la forme et de la dimension du pédoncule destiné à l'emmanchement... ? Certaines variations tiennent peut-être à des fonctions différentes: rien n'exclut qu'un gibier donné ait nécessité une forme de flèche donnée; d'autres témoignent de l'adaptation à la matière première disponible, parcimonieuse ou abondante, aux caractéristiques physiques ou techniques de celle-ci, médiocre ou de qualité; d'autres encore à la dextérité de l'artisan; d'autres enfin à des préférences stylistiques. Tout cela ne diffère pas de ce que nous avons dit à propos du biface ou de la cuillère touarègue, mais tout y est si emmêlé qu'il est difficile de faire la part de ce qui revient à telle cause plutôt qu'à telle autre ${ }^{29}$. L'une des voies possibles pour tenter d'élucider cette question est celle de l'ethno-archéologie. Pierre et Anne-Marie Pétrequin sont parmi les premiers à avoir développé ce type de démarche. Pour mieux comprendre les techniques de fabrication et d'utilisation des haches en pierre polie abandonnées en Europe depuis le Chalcolithique, ils ont entrepris une étude complète des haches fabriquées et utilisées encore aujourd'hui en Nouvelle-Guinée. Outre les nombreuses informations techniques que cette observation a pu leur apporter, elle a enrichi la réflexion concernant la variabilité des outils et permis de remettre en question certaines intuitions. Ainsi, la corrélation entre la valeur d'un objet et son aspect peut être différente de ce que suggère une intuition non prévenue, certaines haches, plus sommaires, moins résistantes, moins bien polies, jouant en réalité un rôle cultuel et ayant une grande valeur symbolique (Pétrequin et Pétrequin 1993). D'autres études ethno-archéologiques menées sur la céramique ont montré que la variabilité individuelle pouvait être très forte, par exemple dans un ensemble de pots issus de la fournée d'un potier, contrairement aux postulats archéologiques (Miller 1985; Laubenheimer et Arcellin-Pradelle 1985, cité par Demoule 2005).

Quant à l'autre objet que j'ai considéré, la cuillère, elle avait une fonction simple. Mais la notion même de fonction est en réalité plus complexe que Leroi-Gourhan ne l'avait soupçonné. Ainsi, il donne l'exemple de la dague, qui doit avoir une lame de 30 à $40 \mathrm{~cm}$ avec une partie percutante très aiguë de section carrée ou losangique de façon à pouvoir percer une cotte de maille ou les joints d'une armure. Pour lui, les différences entre les dagues existantes marquent la personnalité globale du groupe. Pour reprendre ses termes, "la même fonction peut, d'une culture à l'autre, se draper dans des formes équivalentes quoique fortement marquées par la personnalité globale du groupe» (Leroi-Gourhan 1965: 130). Les propriétés des dagues de trois grandes régions du monde (Europe, Proche-Orient, Japon) sont les mêmes (qualité de l'acier, pénétration des pointes) mais elles présentent des différences de formes avec, en Europe, des épées courtes à double tranchant, au Proche-Orient, des dagues en forme de couteau droit, et, au Japon, des dagues courbes proches d'un sabre court. Leroi-Gourhan note qu'il est probable qu'aucune de ces dagues «ne répond tout à fait à l'idéal théorique de pénétration, et il est nécessaire de recourir à la notion d'approximation fonctionnelle pour caractériser une réponse aux exigences contradictoires de satisfaction mécanique et d'empreinte du milieu intérieur du groupe" (ibid.). II me semble avoir sous-estimé ici le fait que, s'il s'agit dans les trois cas de percer une surface résistante - armure, cuirasse ou cotte de maille -, il y a peu de chance que le geste ait été le même. On ne manie pas une lame courbe comme une lame droite, et le geste doit certainement 
différer aussi si la lame possède deux tranchants comme l'épée ou un seul comme le couteau. On peut reprendre ici la distinction qu'opèrent certains auteurs, comme Patrice Flichy pour les machines et Sigaut pour les outils, entre fonction (ou usage) et fonctionnement. La fonction d'un outil est décrite par la réponse à la question: à quoi sert-il? Son fonctionnement est décrit par la réponse à la question: comment s'en sert-on (Flichy 1995: 124 sq.; Sigaut 1991)? Nos trois armes ont donc sans doute le même usage, mais leur fonctionnement, c'est-à-dire la façon dont on leur fait réaliser leur fonction, est différent dans chaque cas ${ }^{30}$.

S'il y a variation culturelle, ce n'est pas tant dans la forme des objets que dans la façon dont on a choisi de réaliser une fonction donnée. Percer une armure est un geste technique, qu'on peut envisager comme tel. Faire la guerre est une autre affaire, bien plus complexe, qui suppose aussi un art qui lui est propre. Lorsqu'on pratique l'art de la guerre, on perce sans doute des armures à l'occasion, avec des armes conçues pour le faire de la façon la plus adéquate possible, mais aussi avec des gestes dont la maîtrise constitue une des composantes de cet art. Encore un point que Leroi-Gourhan semble avoir sous-estimé. II me semble que les ethnologues qui, surpris que deux peuples voisins puissent avoir des outils différents, ne trouvent pas d'autre raison à cela que le souci de se singulariser l'ont sous-estimé aussi. Si des outils sont différents, ce peut être parce que, bien qu'ayant la même fonction, ils ne fonctionnent pas de la même manière. Les gestes nécessaires à ce fonctionnement doivent s'apprendre, et on peut dire de cet apprentissage tout ce qu'on a dit des gestes que requiert la fabrication des outils.

Ici s'achève mon parcours. II m'a parfois mené assez loin du biface, mais seulement dans la mesure où la discussion des thèses de Leroi-Gourhan me l'imposait. Car c'est l'intériorité des hommes et des femmes de la préhistoire qui m'intéressait en premier lieu. Elle est hors de notre portée et on ne saura jamais ce qu'était leur sentiment du beau. J'ai simplement voulu ici faire partager ma conviction, acquise dans la fréquentation des objets qu'ils nous ont laissés - bifaces à la symétrie si parfaite, mais aussi meules et molettes usées par des gestes mille fois répétés ${ }^{31}$-, que, de leur temps déjà, la beauté était présente dans le travail de leurs corps et l'œuvre de leurs mains ${ }^{32}$.

Université Jean-Moulin-Lyon III Archéologies et sciences de l'Antiquité, Nanterre sophie.de-beaune@mae.cnrs.fr
30. Je me permets de signaler ici que j'ai moi-même repris cette distinction, qui permet d'affiner les niveaux d'analyse en préhistoire (Beaune 2000).

31. Beaune 2000 .

32. D'après John Locke, cité par Arendt 1961 (1958) : 123.

\section{remerciements}

Je remercie Pierre Lemonnier et Thierry Bonnot pour leur lecture attentive et leurs suggestions, qui m'ont permis d'améliorer une première version de ce texte. 
De la beauté du geste technique en préhistoire. Par Sophie A. de Beaune

\section{Arendt, Hannah}

1961 (1958) Condition de l'homme moderne. Paris, Calmann-Lévy.

\section{Arnold, Dean E.}

1981 «A model for the identification of non-local ceramic distribution. View from the present ", in Hilary Howard et Elaine Morris (dir.), Production and Distribution. A Ceramic Viewpoint. Oxford, Archaeopress ("British Archaeological Report International Series » 120): $31-44$

\section{Beaune, Sophie A. de}

1999 (1995) Les Hommes au temps de Lascaux. 40 000-10 000 avant J.-C Paris, Hachette ("La vie quotidienne. Civilisations et sociétés»).

2000 Pour une archéologie du geste. Broyer, moudre, piler, des premiers chasseurs aux premiers agriculteurs. Paris, CNRS Éditions.

2008 L'Homme et l'outil. L'invention technique durant la préhistoire. Paris, CNRS Éditions ("Le passé recomposé").

2009 «Technical invention in the Palaeolithic: what if the explanation comes fromthe cognitive and neuropsychological sciences? ", in Sophie A. de Beaune, Frederick L. Coolidge et Thomas Wynn (dir.), Cognitive Archaeology and Human evolution.New York-Cambridge, Cambridge University Press: 3-14.

2011 «L'émergence des capacités cognitives chez l'homme.

I, Les premiers homininés.

II, Les Néandertaliens.

III, Le processus de l'invention: approche cognitive ", in René Treuil (dir.), L'Archéologie cognitive. Paris, Éditions de la Maison des sciences de l'homme: I, 33-90.

\section{Beaune, Sophie A. de}

(dir.)

2013 (2007) Chasseurs-cueilleurs. Comment vivaient nos ancêtres du Paléolithique supérieur. Méthodes d'analyse et d'interprétation en préhistoire. Paris, CNRS Éditions.

\section{Beaune, Sophie A. de et Hilaire-Pérez, Liliane}

2012 Dossier «Esthétique de la technique ", Revue de synthèse $133(6 / 4): 471-556$

\section{Bidet, Alexandra}

2007 "Le corps, le rythme et l'esthétique sociale chez André Leroi-Gourhan ", Techniques \& Culture 48-49: 15-38.

\section{Boas, Franz \\ 2003 (1927) L'Art primitif. \\ Paris, Adam Biro.}

\section{Bordes, François}

1961 Typologie du Paléolithique ancien et moyen. Bordeaux, Publications de l'Institut de préhistoire de l'université de Bordeaux, imprimerie Delmas.

\section{Bromberger, Christian}

1973 «Ethnologie, linguistique, esthétique. Note sur le "style ethnique " ", in L'Homme hier et aujourd'hui, recueil d'études en hommage à André Leroi-Gourhan. Paris, Cujas: $263-278$.

\section{Brumm, Adam et Moore, Mark W.}

2012 «Biface distributions and the Movius Line: A Southeast Asian perspective", Australian Archaeology 74 : 32-46.

\section{Camps, Gabriel}

1979 Manuel de recherche préhistorique. Paris, Doin.

\section{Coquet, Michèle}

2012 "Voir, sentir, figurer », L'Homme 203-204 : 429-456.

\section{David, Johan}

1997 L'Outil. Turnhout, Brepols ("Typologie des sources du Moyen Âge occidental » 78).

\section{Deforge, Yves}

1994 "L'évolution des objets techniques », in Gilbert Simondon, Une pensée de l'individuation et de la technique. Paris, Albin Michel («Bibliothèque du Collège international de philosophie») : 173-181.

\section{Demoule, Jean-Paul}

2005 "Archéologie, style et société», in Bruno Martinelli (dir.), L'Interrogation du style. Anthropologie, technique et esthétique. Aix-en-Provence, Presses universitaires de Provence: 49-66.

\section{Flichy, Patrice}

1995 L'Innovation technique. Récents développements en sciences sociales. Vers une nouvelle théorie de l'innovation. Paris, La Découverte ( Sciences et société »).

\section{Gärdenfors, Peter}

2007 (2004) Comment Homo est devenu sapiens. Auxerre, Sciences Humaines.

\section{Gell, Alfred}

1998 Art and Agency. An Anthropological Theory.

Oxford, Clarendon Press.

\section{Gosselain, Olivier P.}

2012 «D'une histoire à l'autre. Retour sur une théorie des liens entre langues et techniques en Afrique ", in Nathan Schlanger et Anne-Christine Taylor (dir.) La Préhistoire des autres.

Perspectives archéologiques et anthropologiques. Paris,

La Découverte-Inrap: 83-98.

\section{Graves-Brown, Paul}

2000 Matter, Materiality and Modern Culture. Londres, Routledge.

\section{Haudricourt, André-Georges}

2010 "Essai sur les techniques dans les sociétés pré-machinistes ", in Des gestes et des techniques, présenté et commenté par Jean-François Bert. Paris, Éditions de la Maison des sciences de l'homme-Quae: 23-160.

\section{Hilaire-Pérez, Liliane}

2000 L'Invention technique au Siècle des lumières. Paris, Albin Michel.

\section{Hodder, lan}

1982 Symbols in Action Ethnoarchaeological Studies of Material Culture. Cambridge, Cambridge University Press.

\section{Laubenheimer, Fanette et Arcellin-Pradelle, Charlette}

1985 «La notion de série en céramique tournée», in Histoire des techniques et sources documentaires, actes du colloque du GIS, cahier $n^{\circ} 7$. Aix-en-Provence, Maison de la Méditerranée, CNRS et université de Provence: 129-139.

\section{Lemonnier, Pierre}

1991 «De la culture matérielle à la culture? Ethnologie des techniques et préhistoire", in 25 ans d'études technologiques en préhistoire. Bilan et perspectives, Rencontres internationales d'archéologie et d'histoire d'Antibes, 1990. Juan-les-Pins, Apdca: 15-20.

\section{Lemonnier, Pierre (éd.)}

1993 Technological Choices. Transformation in material cultures since the Neolithic. Londres-New York, Routledge.

\section{Leroi-Gourhan, André}

1965 Le Geste et la Parole. II, La Mémoire et les Rythmes. Paris, Albin Michel.

1968 "L'expérience ethnologique " in Jean Poirier (éd.), Ethnologie générale. Paris, Gallimard ("Encyclopédie de La Pléiade ») : 1816-1825.

1971 (1943) L'Homme et la Matière. Paris, Albin Michel.

\section{Lumley, Henry de et al.}

2008 "L'industrie du Paléolithique inférieur du site de l'homme de Yunxian ", in Henry de Lumley et Li Tianyuan (dir.), Le Site de I'homme de Yunxian, Quyuanhekou, Quingqu, Yunxian, province du Hubei. Paris, CNRS Éditions: 467-583.

\section{Machin, Anna J., Hosfield, Robert T. et Mithen, Steven J.}

2007 "Why are some handaxes symmetrical? Testing the influence of handaxe morphology on butchery effectiveness ", Journal of Archaeological Science 34 : 883-893. 


\section{Mahias, Marie-Claude}

1994 «Façonnage de l'argile et de la société en Inde. Une approche ethnologique des techniques ", in Bruno Latour et Pierre Lemonnier (dir.), De la préhistoire aux missiles balistiques. L'intelligence sociale des techniques. Paris, La Découverte («Recherches»): 187-201.

\section{Marchand, Grégor}

2000 «Facteurs de variabilité des systèmes techniques lithiques au Mésolithique récent et final dans l'ouest de la France ", in Pierre Crotti (éd.), Méso '97, actes de la table ronde "Épipaléolithique et Mésolithique », Lausanne, 21-23 novembre 1997. Cahiers d'archéologie romande 81 37-47.

\section{Martinelli, Bruno}

2005 «Style, technique et esthétique en anthropologie ", in Bruno Martinelli (dir.), L'Interrogation du style. Anthropologie, technique et esthétique. Aix-en-Provence, Presses universitaires de Provence: 19-48.

\section{Martinelli, Bruno (dir.)}

1994 Atouts et outils de l'ethnologie des techniques, actes du colloque "Sens et tendance en technologie comparée", 16-17 novembre 1992, Techniques \& Culture 21

\section{Mauss, Marcel}

1967 (1947) Manuel d'ethnographie, préface de Denise Paulme. Paris, Payot.

\section{Mille, Benoît}

\section{et Darblade, Maria-Pia}

2012 «Le pied colossal de bronze de Clermont-Ferrand et la question de l'atelier de Zénodore ", in Martine Denoyelle, Sophie Descamps, Benoît Mille et Stéphane Verger (éd.), Bronzes grecs et romains, recherches récentes, actes du colloque organisé en hommage à Claude Rolley, INHA, 16-17 juin 2009. Paris, INHA «Collections électroniques »), http://inha.revues. org/3909.

\section{Miller, Daniel}

1985 Artefacts as Categories, Cambridge, Cambridge University Press.

\section{Pelegrin, Jacques}

2009 "Cognition and the emergence of language: A contribution from lithic technology ", in Sophie A. de Beaune, Frederick L. Coolidge et Thomas Wynn (éd.), Cognitive Archaeology and Human Evolution. Cambridge, Cambridge University Press: 95-108.

\section{Pétrequin, Pierre} et Pétrequin, Anne-Marie

1993 Écologie d'un outil : la hache de pierre en Irian Jaya (Indonésie). Paris, CNRS Éditions ("Monographie du Centre de recherches archéologiques"12).

\section{Piette, Édouard}

1889 «L'art pendant l'âge du renne; la question de la domestication du renne", Congrès international d'anthropologie et d'archéologie préhistoriques, $x^{\oplus}$ session, Paris, 1889: 159-161.

\section{Potts, Rick}

1994 «Variables versus models of early Pleistocene hominid land use ", Journal of Human Evolution 27: 7-24.

\section{Preston, Beth}

2000 "The functions of things", in Paul Graves-Brown (éd.), Matter, Materiality and Modern Culture. Londres, Routledge : 22-49.

\section{Sackett, James R.}

1990 "Style and ethnicity in archaeology: The case for isochrestism ", in Margaret W. Conkey et Christine A. Hastorf (éd.), The Uses of Style in Archaeology. Cambridge, Cambridge University Press ( New Directions in Archaeology ") : 32-43.

\section{Sahlins, Marshall}

1972 Âge de pierre

âge d'abondance. L'économie des sociétés primitives. Paris, Gallimard.

1980 (1976) Au cœur des sociétés. Raison utilitaire et raison pratique. Paris, Gallimard ("Bibliothèque des sciences humaines»).

\section{Saint-Exupéry, Antoine de}

1939 Terre des hommes.

Paris, Gallimard.

\section{Schick, Kathy D. et Toth, Nicholas}

1993 Making Silent Stones Speak: Human Evolution and the Dawn of Technology. Londres, Phoenix.

\section{Sigaut, François}

1991 "Un couteau ne sert pas à couper, mais en coupant. Structure, fonctionnement et fonction dans l'analyse des objets ", in 25 ans d'études technologiques en préhistoire. Bilan et perspectives, Rencontres internationales d'archéologie et d'histoire d'Antibes, 1990. Juan-les-Pins, Apdca: 21-34.

2012 Comment Homo devint faber Paris, CNRS Éditions ("Le Passé recomposé»).

\section{Simondon, Gilbert}

1958 Du mode d'existence des objets techniques. Paris, Aubier.

\section{Yamei, Hou et al.}

2000 «Mid-Pleistocene

Acheulean-like stone technology of the Bose basin, South China ", Science 287(5458) : 1622-1626.

\section{Zumthor, Paul}

1993 La Mesure du monde. Représentation de l'espace au Moyen Âge. Paris, Seuil ("Poétique»). page 26 et ci-contre Série de bifaces des vallées de la Somme, de l'Oise et de l'Aisne découverte fin $x x^{\ominus}$. début $x x^{\ominus}$, Paléolithique inférieur et moyen $(300000$ à 40000 ans av. J.-C.). @ Jean Luc Katzman (www.aggsbach.de) 